\title{
Classification of NFRs for Information System
}

\author{
Yahiya Gazi \\ M. Tech. Scholar, \\ Department of Computer \\ Science and \\ Engineering, Al-Falah School \\ of \\ Engineering and Technology, \\ Dhauj, Faridabad, Haryana, \\ India
}

\author{
M. Sarosh Umar \\ Department of Computer \\ Engineering \\ Aligarh Muslim University, \\ Aligarh-202001, U.P, India
}

\author{
Mohd. Sadiq \\ Computer Engineering \\ Section, UPFET, Jamia Millia \\ Islamia (A Central \\ University), \\ New Delhi-110025, India
}

\begin{abstract}
Software requirements are broadly classified into two parts, i.e., functional requirements (FR) and non-functional requirements (NFRs). In recent year, NFRs have received much attention by requirements engineering community. This can be verified from the importance of the NFR framework. This framework was developed to elicit and model only the NFRs. There are different NFRs for different types of system like information system, web based system, real time system etc. Elicitation of NFRs for these types of systems is an important research issue. Therefore, the objective of this paper is to classify various NFRs for Information System so that requirements analyst can easily identify the complete list of NFRs according to their need in early phase of requirements engineering.
\end{abstract}

\section{Keywords}

Requirements Engineering, Non Functional Requirements, Quality Requirements, SIG.

\section{INTRODUCTION}

Non-functional requirements (NFR) explain essential constraints upon the development of the proposed system. They identify a wide range of qualities such as security requirements, performance requirements, availability requirements, interoperability requirements, viability requirements and portability requirements; and these qualities play a key role in driving architectural design [1]. At the time of requirement elicitation, NFR may be conflicted with each other. For example, accuracy and performance are conflicted NFRs [5].

In literature, we have identified some classification schemes of NFRs [5, 6], for example, ISO/IEC 9126 classified NFRs into four levels, i.e., "quality in use, external quality, internal quality and process quality". Existing classification schemes $[2,3,6]$ do not provide non functional requirements taxonomy according to the type of system like information system, web based system real time system etc. For example, at the time of requirements elicitation, it is important to know that, how many NFR would be there if we want to develop different types of systems? Therefore, it motivates us to present the classification schemes of NFR for information system development only.

This paper is organized as follows: In section II we discuss the literature review of NFRs. Section III presents classification of NFRs.In section IV, we explain how the proposed classification of NFRs is important at the time of requirements elicitation. Conclusion and future work are given in section $\mathrm{V}$.

\section{LITERATURE REVIEW}

This section presents a literature review of NFRs. In literature, we have identified different classification scheme of NFRs. For example, the standard ISO/IES 9126 [3] distinguish 4 types of quality levels. Peach et al. [9] provides the following process oriented classification: (i) "The identification of NFR from different viewpoints and different levels of detail", (ii) "The support for uncovering dependencies and conflict between them, and discuss and prioritize them accordingly", (iii) "The documentation of NFR and the evaluation of this documentation", (iv) "The support for identifying means to satisfy the NFR, to evaluate and discuss means, and to make trade -off decision according this includes cost estimation", (v) "The support for change and project management.". In [6] we identify another classification based on the concept of NFR framework [7] proposed by Jureta et al [4] "This scheme was based on "goals and softgoals", driven by the non behavioral perspective". This classification includes four categories of goals: functional hardgoals, non functional hard goals, functional soft goals and nonfunctional soft goals.[10]In Roman presents the following classification scheme.

Interface requirements explain "how the system is to interface with its environment, users and other systems". For example, user-friendliness (i.e., user interface and their qualities).

Performance requirements explain about the performance constraints include the following "(i) time and /or space bounds, like response time, throughput, workloads and available storage space .e.g., system must handle 150 transactions / second" (ii) reliability, i.e., "system must have less than 1.5 hour download time/5 months.", (iii) security such as permissible information flows, (iv) survivability, such as system endurance under fire, natural catastrophes. Operating requirements include skill level consideration system accessibility for maintenance, physical constraints (size, weight), and personnel availability, etc [10].

Lifecycle requirements can be classified into two sub categories, i.e., (i) design quality ; and it includes the following like "maintainability, enhanceability, portability", and "(ii) restrictions on development like "resource availability", " methodological standards" ," development time limitations" etc. 
Economic requirements include immediate and/or long term costs and Political requirements.

Boehm et al [16] proposed a classification of NFRs on the basis of software quality tree. This is further sub-classified as portability, as-is utility, and maintainability. Portability includes device-independence and self-contentedness; asis-utility is decomposes into reliability, efficiency, and human engineering; Maintainability includes following types of NFRs like testability, understandability, and modifiability.

Hewlett-Packard presented another classification using software quality attributes and NFRs, i.e., FURPS. This classification scheme was extended by [4], and it is referred to as FURPS +. FURPS stands for "Functionality, Usability, Reliability, Performance, and Supportability". Important attributes of these NFRs as given as below [4]:

Functionality: Attributes includes "feature set, capabilities, generality, and security".

Usability: Attributes includes "human factors, aesthetics, consistency, and documentation".

Reliability: Attributes includes "frequency/severity of failure, recoverability, predictability, accuracy, and means time to failure".

Performance: Attributes includes "speed efficiency, resource consumption, throughput, and response time".

Supportability: Attributes includes "testability, extensibility, adaptability, maintainability, compatibility, configurability, installability, localizability, and portability".

In literature, we have identified that "The IEEE-Standard 830 - 1993" lists 13 non-functional requirements: Performance requirements, Security requirements, Accuracy requirements, Verifiability requirements, Confidentiality, requirements, Reliability requirements, Maintainability requirements, Privacy requirements, Usability requirements, Provability requirements, Reusability Requirements, Inter-operability requirements, Traceability requirements, Viability requirements, and Standardizability requirements.

\section{PROPOSED CLASSIFICATION}

In this section, we present our classification scheme of NFRs. In the proposed classification, we classify the NFRs on the basis of the following criteria, i.e., important NFRs for Information System, NFRs similar to real time systems, NFRs similar to web based system, safety critical system, and real time system. A tree like structure of the proposed classification scheme is given in Fig. 1.
(A) Important NFRs for Information System (IS) In literature, we have identified some NFRs that are indispensible for the development of every IS like provability, reusability, standardizability, traceability, and viability.

\section{(B) NFRs Similar to Real time systems}

In this criterion, we identified those NFRs of IS which are similar to real time systems like accuracy, confidentiality, and verifiability.

(C) NFRs Similar to Web based systems In this criterion, we identified those NFRs of IS which is similar to web based systems like interoperability, and privacy.

(D) NFRs Similar to Real and wed based systems

In this criterion, we identified those NFRs of IS which is similar to real time systems and web based systems like security, usability, and performance.

\section{CASE STUDY}

In this section we present how the proposed classification of NFRs is useful at the time of requirements elicitation [8, $11,12,13,14,15]$. In our case study, we use an example of Information System (IS).

In our classification schemes of NFRs, Provability, Reusability, Standardizability, Traceability, and Visibility are the actual NFRs of IS. Therefore, these NFRs must be present in the software requirements specification document.

Reliability and Availability are safety critical and processcontrolled system, respectively, NFRs for Information System. From our classification it is clear that Reliability can further be decomposed into Completeness, Accuracy, Maturity, and Compliance because these are the important attributes of Reliability.

Accuracy, Confidentiality, and Verifiability are the real time system NFR. These NFRs must be elicited during early phase of requirements engineering. Interoperability and Privacy are the important NFRs which are similar to Web based system. Security, Usability and Performance are the most important NFRs of IS, Real time system, and Web based system. At the time of decomposing and refining the Security NFR, it can be further decomposed into Confidentiality, Availability, Access Control, Authentication, and Integrity. Usability can further be divided into Learnability, Operability, Ease of Use, Usefulness, and Productivity. Similarly, Performance can further be decomposed into the following sub-NFRs like Response-Time, Space, capacity, Latency, Throughput. 


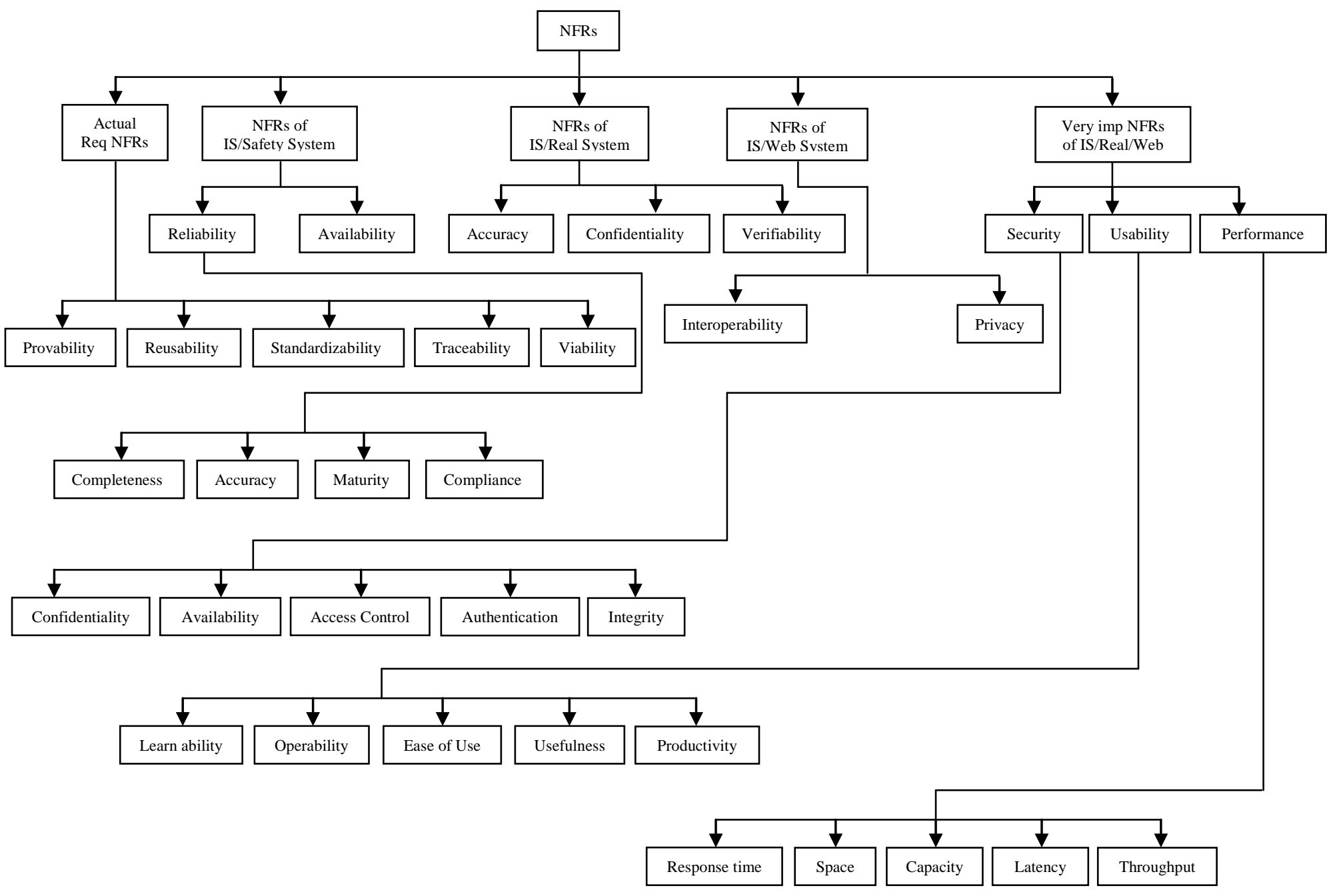

Fig. 1 Classification of NFRs for IS

\section{CONCLUSION AND FUTURE WORK}

In this paper, we have classified NFRs for IS on the basis of actual NFRs; NFRs similar to safety critical and process controlled system; NFRs similar to real time system; and NFRs similar to Web based system. Such type of classification would be useful to elicit the NFRs at the time of requirements elicitation of IS. In future we will try to work on the following issues. The future scope of this classification in any information system to classify based on these NFRs which can make system fast and reliable.

1. Analysis of NFRs using StarUML

2. Prioritization of NFRs using TOPSIS method

\section{REFERENCES}

[1] Chung L., Leite JCSP "On Non-Functional Requirements in Software Engineering," A.T Borgida et al. (Eds): Mylopoulos Festschrif, LNCS 5600, pp.363-379, Springer-VerlagBerlin Heidelberg 2009.

[2] Grady, R., Caswell, D.: Software Metrics: Establishing a Company-wide Program. Prentice-Hall, Englewood Cliffs 1987.

[3] ISO/IEC 9126-1:2001(E): Software Engineering Product Quality - Part 1: Quality Model 2001.

[4] Jureta, I.J., Faulkner, S., Schobbens, P.-Y.: A more expressive softgoal conceptualization for quality requirements analysis. In: Embley, D.W., Olivé, A., Ram, S. (eds.) ER, LNCS, Vol. 4215, pp. 281-295. Springer, Heidelberg 2006.

[5] Mairiza Dewi and Zowghi Didar, "Constructing a Catalogue of conflicts among Non-functional requirements," L.A Maciaszek and P. Loucopoulos (Eds.):ENASE 2010, CCIS 230, pp.31-44, 2011@Springer-Verlag Berlin Heidelberg 2011.

[6] Mairiza Dewi, Zowghi Didar, Nurmuliani Nurie "An Investigation into the Notion on Non-functional Requirements," SAC10 pp. 22-26, Sierre Switzerlandm, March 2010.

[7] Mylopoulos, J., Chung, L., Nixon, B.: Representing and Using Nonfunctional Requirements: A Process-Oriented Approach. IEEE Trans. Software Eng. 18(6), pp. 4834971992.

[8] Nuseibeh B, "Weaving together requirements and architecture", IEEE Computer 34(3):, pp. 115-117, 2001.

[9] Paech, B., Kerkow, D.: "Non-Functional Requirements Engineering - Quality is Essential." In: 10th Anniversary International Workshop on Requirements Engineering: Foundationfor Software Quality, REFSQ 2004. 
[10] Roman, G.-C.: “A Taxonomy of Current Issues in Requirements Engineering. IEEE Computer," pp. 14-21 April 1985.

[11] Sadiq M and Jain SK “A Fuzzy Based Approach for the Selection in Goal Oriented Requirements Elicitation Process," International Journal of System Assurance Engineering and Management, Springer, 2014.

[12] Sadiq M and Jain SK "An Insight into Requirements Engineering Processes", International Conference of Advance in Communication, Networking and Computing (CNC), LNCSIT, Springer, pp. 313-318, 2012 India.

[13] Sadiq M and Jain SK "Applying Fuzzy Preference relation for Requirements Prioritization in Goal Oriented Requirements Elicitation Process", International Journal of System Assurance Engineering and Management, Springer Vol. 5 Issue 4 pp. 711-723, 2014.
[14] Sadiq M and Jain SK, "A Fuzzy Based Approach for Requirements Prioritization in Goal Oriented Requirements Elicitation Process", International Conference of Software Engineering and Knowledge Engineering (SEKE), pp. 54-58, 2013 USA.

[15] Sadiq M and Jain SK, "Stakeholders Identification Methods in Goal Oriented Requirements Elicitation Process", Fifth IEEE International Workshop on Requirements Prioritization and Communication at IEEE International Requirements Engineering Conference (RE), pp. 25-33, 2014 Karlskrona, Sweden.

[16] Boehm, B.W., Brown, J.R., Kaspar, H., Lipow, M., MacLeod, G.J., Merritt, M.J.: Characteristics of Software Quality. North-Holland, Amsterdam 1978. 